\title{
Robots We Like to Live With?! - A Developmental Perspective on a Personalized, Life-Long Robot Companion
}

\author{
Kerstin Dautenhahn \\ Adaptive Systems Research Group, University of Hertfordshire \\ College Lane \\ Hatfield, Herts, AL10 9AB, United Kingdom \\ E-mail K.Dautenhahn@herts.ac.uk
}

\begin{abstract}
This paper addresses different possible social relationships between robots and humans, drawing on animal-human relationships. I will argue that humans have been living in (generally peaceful) co-existence with a number of potentially dangerous species, such as some canines. Interestingly dogs are not born 'pet dogs', it's not completely 'predefined' in their genes whether they will become friendly or dangerous. A critical period in a puppy's early life significantly shapes its socialization and behavioral conformation. I will suggest that such a developmental model of socialization could be an interesting viewpoint on the design of future generations of robots that need to co-exist with humans, and that humans like to live with. I will propose the challenge of developing 'personalized robot companions', machines that can serve as life-long companions. I will argue that such individualized robots are necessary due to human nature: people have individual needs, likes and dislikes, preferences and personalities that a companion would have to adapt to: one and the same robot will not fit all people. Cognitive robot companions above all need to be socialized and personalized in order to meet the social, emotional and cognitive needs of people they are 'living with'.
\end{abstract}

\section{Introduction: Socializing Robots}

Application areas for robots that heavily involve human contact are a particularly challenging domain. The European Integrated Project Cogniron (www.cogniron.org) is a recent example of an endeavor to develop a cognitive robot companion. Interaction and communication of embodied physical robots with humans is multimodal, and involves deep issues of social intelligence and interaction that have traditionally been studied e.g. in psychology. The design of a robot's behavior, appearance, and cognitive and social skills is scientifically highly challenging, and requires interdisciplinary collaborations across the traditional boundaries of established disciplines. Initial contact often relies on anthropomorphic attribution and the novelty effect, which has problematic consequences for long-term and/or repeated interaction with robots: the novelty effect wears out quickly $[1,2]$, and anthropomorphism might raise false expectations regarding the cognitive and social abilities that the robot cannot fulfill. Likewise, robots that mimic as closely as possible human appearance and behavior are not necessarily the optimal path towards social robots $[4,5,6]$, (cf. the Like-Like Agents Hypothesis, [3]).

It is at present not quite clear what roles robots will adopt [7]. Will they be effective machines performing tasks on our behalf, assistants, companions, or even friends? What social skills are desirable and necessary for such robots to be believable and acceptable to humans? People have often used technology very differently from what the designers originally envisaged, so the development of robots designed to interact with people requires a careful analysis and study of the requirements.

In this paper I will argue that rather than relying on an inbuilt fixed repertoire of social behaviors, a robot should be able to learn and adapt to the social manners, routines and personal preferences of the people it is living with. Many developmental approaches towards robotics have focused on models inspired by human psychology, e.g. models proposed by Piaget or Vygotsky that have greatly influenced the field of developmental and epigenetic robotics $[8,9]$. However, in the domain of social development, humans are not the only suitable model. While many robotics approaches aim to achieve humanlike cognitive abilities in robots, there is one mammal that can serve as an interesting model of a 'companion': the dog. Dogs can't read, can't write, can't play chess and generally are not able to perform tasks involving complex language skills, manipulation, or problem-solving. However, dogs have proven to be ideal life-long companions for many people all over the globe. They give company, provide emotional support, and even perform a variety of tasks, e.g. as guard dogs, as guides for the blind, some even serve as seizure alert dogs (the mechanisms employed in the latter are still widely unknown). Not surprisingly, researchers have recognized the potential of 
dogs as models for interactive characters or pet robots that can be trained similar to a dog [10,11]. In this paper, I propose to consider in more depth the developmental trajectory that can transform a dog puppy into a valued member of our household, a process that $I$ argue can be beneficial to 'growing up robots' [12].

A puppy that grows up in a family quickly learns the 'rules of the house', it learns about its position in the family hierarchy, it learns to distinguish and recognize individual family members, and it will respond with great sensitivity to any emotional and social cues. When suitably trained and exposed to the right (social) environment it will learn to 'fit in'. However, a dog's socialization does not unfold automatically due to a genetically fixed schedule: the period of socialization is critical and will determine the social abilities of the adult dog. This critical period will determine the dog's behavioral conformation, its social personality which is vital for its position in the house, and the extent to which its behavior can be controlled by its human companions. In the next section I discuss the issue of predictability and control in human-machine interaction. Next I present some work on dog socialization and outline implications for robotics research. I then discuss the need to develop personalized robots, in order to achieve the goal of lifelong robotic companions that we would like to live with.

\section{Growing up Robots}

\section{$2.1 \quad$ Control and predictability}

In human-computer interaction issues of predictability and control have been widely discussed. Pattie Maes advocates strongly the autonomous agents - or indirect management approach, agents that on our behalf do their jobs with little human intervention and thus free us from workload [13]: "The metaphor used is that of a personal assistant... who becomes gradually more effective as it learns the user's interests, habits, and preferences (as well as those of his or her community)." (p. 145). Ben Shneiderman on the other hand strongly argues against agents as a human-machine paradigm, since they take control away from the human, and makes the computer unpredictable. He advocates the interface metaphor of direct manipulation, rather than delegation [14], his vision are predictable and controllable user interfaces that users can manipulate with ease. In light of the discussions above, let us reformulate the Maes versus Shneiderman debate into: Do we want robots that are under our total direct control with interfaces that can directly manipulate the robot? Or should they be widely autonomous, with their own agendas, robots that are not under our total control but that can be trained, robots that get to know us, that we can live with and that can live with us? A continuum of design choices, with different choices appropriate for different applications can be considered [15].

For thousands of years human culture has developed different types of inter-species relationships, relationships with other animals, animals that can potentially be dangerous for humans to live with but that become welcome additions to our household. Such relationships provide a huge source of inspiration as to how machinehuman relationships and the role of machines in our society might develop, advocating a future where robots can be 'domesticated' and exist as members of our families ${ }^{l}$. The next section provides a little excursion into the world of dog evolution, breeding, and socialization, based on [16]. I will point out why dog socialization might provide a good model for developing personalized robot companions.

\subsection{Socialization of dogs}

Raymond and Lorna Coppinger have provided a fascinating discussion of how dogs have evolved and how they have become working dogs and companions to humans [16]. Interestingly, from an evolutionary point of view, dogs are by far the most successful member of the genus Canis which also includes jackals, coyotes and wolves, they outnumber their relatives by factors of 10 (compared to jackals) or even 1000 (compared to wolves). Thus, dogs have found a successful 'niche' by sharing their lives with humans (Figure 1). And humans have plenty of benefits from this inter-species relationship: dogs guide the blind, search for explosives, rescue people, pull sleds, and can even serve a therapeutic role.

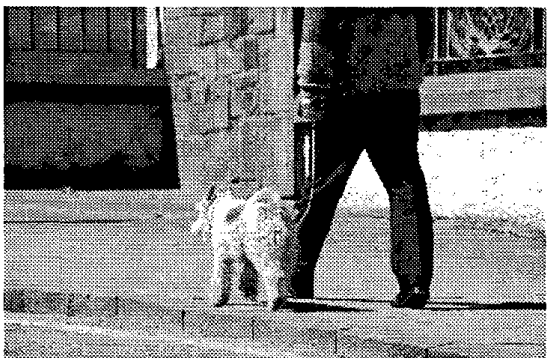

Figure 1: Two members of different species living together in harmony. For many people dogs are truly their best friends. Photo supplied by FreeFoto.com.

A huge variety of different breeds exist, through selective breeding the physical conformation of dogs has been adapted to suit certain tasks. The behavioral conformation of a dog is shaped by a series of developmental environments and events. Here, an important element that

\footnotetext{
${ }^{1}$ Interestingly, this view is in sharp contrast to the scenarios put forward by some prominent Western Artificial Intelligence researchers who predict that robots will one day take over the world, a vision that causes fear and resentment towards robots.
} 
influences a dog's future behavior is a critical period of social development. During this period a puppy has the greatest capacity to learn social skills. During the critical period dominance hierarchies are formed and puppies practice a variety of dominant and submissive behaviors, e.g. they learn to beg for food, whom to beg from, and they learn how to transform begging behavior into social greetings. At about 16 weeks this 'window of opportunity' for social learning closes. Interestingly, the critical period coincides with most of the growth of a dog's brain. Thus, experiences during that critical period will significantly shape the development of behavior patterns that will 'imprint' how a dog will behave in a social situation, what kind of 'social personality' it will have. Later training might modify the dog's personality, but the basic behavioral patterns are 'set for life'. Interestingly, a dog's ontogeny (development of an individual) makes it uniquely suited to become a social being, if growing up in an appropriate social environment.
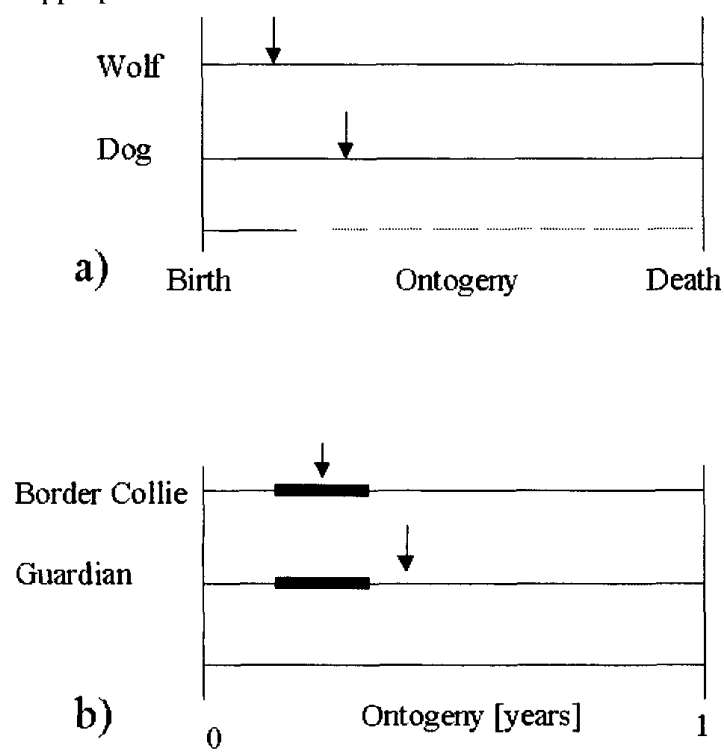

Figure 2: a) Onset of fear (hazard avoidance) behavior in wolves and dogs. b) Onset of foraging (predatory) behavior in border collies and livestock-guardian dogs. Predatory behaviors in guardians only occur after the critical period of social development, predatory behaviors are therefore not included in their social play. The situation is very different for border collies where predatory motor patterns become an inherent part of social play. Bold lines represent the critical periods of social development. Redrawn and modified after [16], pages 107 , 117.

Figure 2a depicts the onset of fear motor patterns in dogs and wolves. It occurs at about 19 days in wolves and 49 days in dogs. Thus, the 'window' for taming a wolf is very small, and if not introduced to people by then he will be difficult to tame. The window of opportunity is much larger for dogs. When a livestock-guardian dog is raised among sheep for the first 16 weeks after birth, then for the rest of his life he will treat sheep as his primary social companions and he will show a variety of interspecific social motor patterns towards them; he will follow sheep, greet them, show dominance and submissive behaviors towards them. The guardian dog will live among the sheep and protect them from predators since during the critical period, when the dog learnt what species he belongs to, he was surrounded by sheep. Thus, from the dog's perspective, he is a sheep among sheep. Similarly, when properly exposed to people during the critical period dogs will grow up as social beings that people like to be with. Dogs tend not to kill other animals if they know them individually (even prey animals), but they also don't kill animals that they grow up with. Breeders use such knowledge of a dog's critical period to shape their behavior, e.g. if foxhounds are exposed to farm livestock during their critical period then they won't hunt them.

Also, individual breeds show different timing in the onset of e.g. predator behavior. As Figure $2 b$ shows predatory motor patterns occur in border collies during the critical period, so e.g. chasing becomes part of their social personality. In livestock-guarding dogs predator behaviors shows only after the critical window of social development has closed, so it won't be part of how they behave socially.

Let us have a closer look at predatory behaviors and how different dog breeds are being tailored (by nature and nurture) to fill particular niches. The 'wild type' of basic predatory motor patterns that e.g. many members of the Canis genus show can be presented as follows ( $>$ are connected motor patterns):

Orient $>>$ eye $>>$ stalk $>>$ chase $>>$ grab-
bite>skill-bite>>dissect

It doesn't mean that each individual will shows this behavior, e.g. as a result of taming during the critical period of socialization a wolf might not show this behavior. But he has genetic predispositions for these predatory behaviors so that if he grows up among wolves, i.e. in his 'natural' (social) environment, he will develop the above mentioned predatory behaviors in a particular sequence, i.e. the individual motor patterns are connected in a particular order. In different breeds of dogs people influenced the sequencing of the above mentioned motor patterns so that that the dogs can be better suited for particular tasks. Emphasizing and rearranging the motor patterns leads to breed-typical motor patterns that make a breed behaviorally unique, i.e. the breed has its own behavioral confirmation [16]. For example, for a hound the sequence looks like: 
orient >>mark>>chase>sgrab-bites>killbite

Pointers typically do not exhibit the stalk, chase, killbite and dissect motor patterns. The best lifestock guarding dogs, when properly raised, will not show any of these predatory behavior patterns. Most will weakly display one or two predatory motor patterns such as chase and grabbite. These examples show that variations of the wild type sequence of predatory motor patterns (see Figure 2 above) can give rise to a variety of different 'social personalities' of dogs.

\subsection{Socialization of Robots}

What are the implications of the previous section for social robots? How can robots be socialized? Note, that while dogs are living, breathing animals that develop from a fertilized egg and are nurtured by their mothers as puppies, robots to date are not made of living material. Researchers working in evolutionary and developmental robotics are trying to bridge this gap, but we are still far from having robots around which in a non-trivial sense show a process of ontogeny shaped by 'nature' and 'nurture'. It is even not very clear what should constitute these two aspects. Similarly, in terms of socialization, what could a 'critical period' mean, why should the period that allows behavioral modification be limited at all? In the following I try to answer some of these questions.

What is nature, what is nurture for robots? Let us assume (hypothetically) that a 'wild type' exists, a robot with a huge behavioral repertoire, a repertoire of motor patterns that can be elicited by certain environmental stimuli, by recollections from memory, by the output of learning algorithms, etc. This wild type shows the 'genetic predispositions', the range of basic behaviors the robot is capable of but that might be modified and added to by learning and adaptation. If such a robot is supposed to live around humans then it needs to know 'how to behave appropriately'. This appropriate behavior could be preprogrammed, but this could not account for the potentially huge design space of social behavior. Also, the designer / programmer would require in-depth knowledge of e.g. of the habits, preferences and 'traditions' within a human family that the robot is supposed to live with. I suggest that shaping the behavioral conformation of a robot to suit its social environment could produce robotic machines that people might like to live with. This would lead to robots with very distinct 'social personalities'. An interesting 'side-effect' of this strategy would allso be that humans probably find such robots more desirable, in the same way as many people prefer to buy a puppy that they can shape rather than an old dog with an already quite firmly established behavior repertoire.

Is it difficult to teach an old robot new tricks? Not necessarily would we want to restrict all learning processes to a critical period. In order to cope with a dynamically changing environment robots need to have life-long learning capacities. Social beings such as dogs or humans, once they exhibit distinct personalities, usually do not change dramatically. A teacher can become a killer, and a guard dog can transform into a loving pet, but more often than not personalities are consistent over an extended period of time. We 'know' what 'kind of person' a friend is, while moods and emotions can change dynamically. Judgments on personality help us to generate certain expectations and make predictions that help guiding and planning our own behavior. Predictions and expectations are powerful means which help us to manage relationships with other people by reducing the need to 're-establish' the personality type of a person at every single encounter. Similarly, it is very likely that robots with personality will be easier to interact with for human beings.

From a Cognitive Science or Artificial Intelligence perspective designing agents with personality is a big challenge [17]. However, so far personality has usually been built-in, specific for a particular application domain and part of the behavioral or cognitive architecture that drives the agent. But socialization of robots as discussed above could provide more, namely personalities that are suited to their social environment, and that might even to a certain degree reflect / mirror the environment they have grow up. It is not difficult to predict the social environment which an angry and very aggressive dog was exposed to early in his life. Similarly, a robot growing up in a caring loving environment is likely to show later in its life rather benign behaviors and interaction styles.

\section{Personalized Life-Long Robot Companions}

Although the design of 'natural interfaces' between humans and machines has been intensively studied, human-machine interfaces often still rely on the ability of humans to adapt to the particular interfaces and interaction styles of machines. For example, compared to humanhuman interaction, there is not much 'naturalness' in how we operate our computers, cars or microwaves. I propose the challenge to develop personalized robots, machines that can serve as companions, and that can adapt to the needs and interaction styles of people they are interacting with, cf. first steps towards this goal in $[18,19]$. Such robots will be individualized, i.e. no two robots will the same. They will also be personalized, i.e. their individuality reflects the needs and requirements of the (social) environment where the robot is operating in. Individualization and personalization are necessary due to human nature: people have individual needs, likes and dislikes, preferences and personalities that a companion would have to adapt to: one and the same robot will not fit 
all people. Similarly, it would be unrealistic to assume that one could design a perfect car model or create a perfect dog breed that will satisfy all people. Robots will have to be individuals, as we are.

The design space of robots in terms of possible behavior repertoires and appearances is vast. It is unlikey that a robot will be able to learn efficiently and in realtime about its social environment 'from scratch'. How we scaffold a robot's social development, how can the life history of a personalized robot look like? I propose the following framework (see Figure 3 ) that consists of different phases:

Phase I: Acquiring background knowledge. Extensive user studies need to reveal how different robot behaviors, as well as appearances influence people's attitudes, opinions and preferences towards robots. The tasks that the robot is supposed to perform, the physical environment that the robot is operating in, as well as the social environment (including expected personality profiles of users) are likely to play an important role. Quantitative as well as qualitative data can lead to a growing body of knowledge that enables us to suggest designs for robots in particular 'niches' [4]. Note, this phase of exploring the design space of social robots, which covers the whole area of humanrobot interaction (HRI), is likely never to be completed.

Phase II: Socialization in the laboratory. Based on the generic knowledge acquired in phase I a first prototype of a robot can be tested and refined under laboratory conditions in order to determine the default settings in its behavioral repertoire (see Figures 3,4). As described in section 2 for dogs, it is here that the robot's 'social personality' will be formed. Later experiences will modify the robot's personality, but the basic behavioral patterns are 'imprinted'. Thus, different 'breeds' of robots can be socialized by 'robot trainers' in controlled environmental conditions. Here, information on the user group (e.g. expected personality profiles) as well as requirements and constraints derived from the particular tasks that the robot is supposed to perform, and the particular type of environment involved, can inform the default settings that need to be determined before the robot is exposed to its naturalistic environment, i.e. placed in a family home. Note, I assume that it is unlikely that people want to spend as much time and effort in socializing a robot as they are willing to invest in a dog. Thus, I assume that customers will prefer if the robot's basic personality traits are being shaped before the robot is being selected. In this way people could select different personality types of robots as they select different dog breeds, according to their preferences and needs.

Phase III: Personalization. Once individual users/families have chosen a particular robot breed, personality profiles and other information that can be acquired about the people and the new home a priori can help to identify the range of likely necessary adjustments of the robot's behavior repertoire. Once the robot is placed in the home, it will interact with the target person or family that it is supposed to live with. It needs to be able to identify and recognize different people, and to know about relationships in the family. In order to become personalized the robot needs to adjust its 'default settings', namely to learn from (life-long) experience. Figure 3 summarizes the proposed model. As a result of this process, even two robots of the same 'breed' that leave the laboratory with identical behavioral default settings, will over time develop into individualized machines, machines with a unique personality. Note, such a robot will have to be able to cope with changes, e.g. changes in the tasks it is supposed to perform, changes in relationships to family members (e.g. if children grow up), etc.

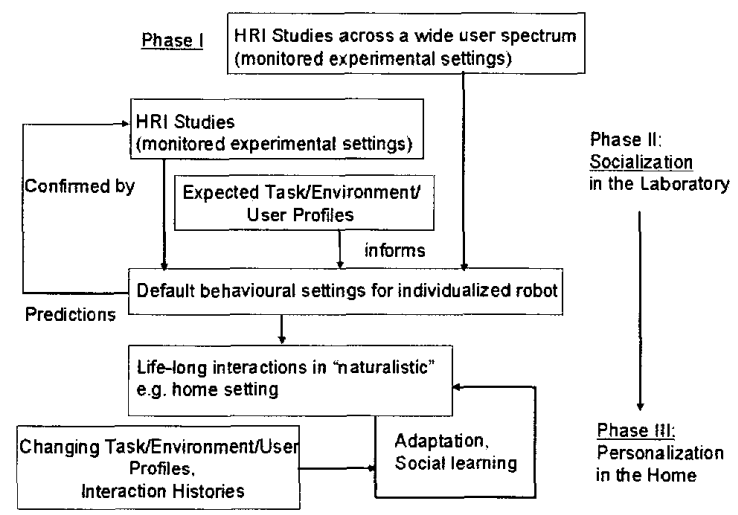

Figure 3: Proposed model of socializing robots: towards personalized robot companions.

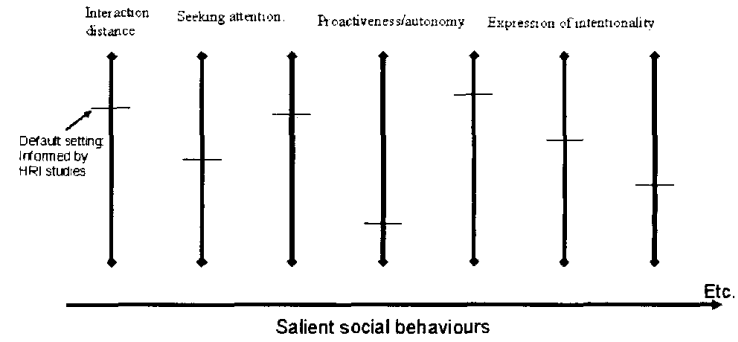

Figure 4: Examples of salient social behaviors that influence human-robot interaction. Various parameters, e.g. regarding the robot's expression of intentionality or autonomy/control, need to be identified in HRI studies.

\section{Conclusion: The Domesticated Robot}

I sketched a framework for robot socialization where in addition to individual and social learning skills that each robot might possess, behavioral conformations are 
achieved by growing up robots in a social environment and using the concept of a critical period to shape robot personalities.

Note, a robot's behavioral and physical confirmations (behavior and appearance) are not independent from each other and need to match the particular 'niche' that the robot has been developed for [4]. Similarly, a Chihuahua or a Pekingese will never become a sled dog due to their physical conformation which does not suit this task.

The ideas on robot socialization that we discussed in this paper can be seen as the developmental dimension of interaction-aware robots [20]: social personalities that are shaped by early experiences in a critical period. Such an approach might affect whether robots can be 'tamed', and whether they will become our best friends or worst enemies. It will be up to us to make these choices. Thus, although we can not directly control the behavior of robots, we can show social control, similar to how we influence and shape the behavior of our children, although we can never completely control them. Socialized robots of this kind could 'naturally' be social; we would not need any artificially imposed rules such as Isaac Asimov's laws. The robots I envisage would 'voluntarily' try to get along with us, because we are part of their social environment. They might ultimately even begin to like us.

Acknowledgements. This work has been partially supported by the European Integrated Project Cogniron (Cogniron robot companion, contract number FP6-IST002020).

\section{References}

[1] T. Kanda, T. Hirano, D. Eaton, H. Ishiguro, "Interactive robots as social partners and peer tutors for children: A field trial", Human Computer Interaction, 19, I-2, pp. 61-84, 2004.

[2] T. Salter, K. Dautenhahn, R. te Boekhorst, "Robots moving out of the laboratory - Detecting interaction levels and human contact in noisy school environments", Proc. Ro-man, 2004.

[3] K. Dautenhahn, "The Art of Designing Socially Intelligent Agents - Science, Fiction, and the Human in the Loop", Applied Artificial Intelligence, Vol 12, 7-8, pp. 573-617, 1998.

[4] K. Dautenhahn, "Design spaces and niche spaces of believable social robots", Proc. Ro-man2002, 192-197.

[5] S. Woods, K. Dautenhahn, J. Schultz, "The design space of robots - Investigating children's views", Proc. Ro-man 2004.

[6] K. Dautenhahn, A Bond, L Cañamero, B Edmonds (eds.), "Socially Intelligent Agents - Creating Relationships with Computers and Robots", Kluwer Academic Publishers, 2002.
[7] K. Dautenhahn, "Roles of robots in human society Implications from research in autism therapy", Robotica, 21, pp. 443-452, 2003.

[8] J. Weng, J. McClelland, A. Pentland, O. Sporns, I. Stockman, M. Sur, E. Thelen, "Autonomous mental development by robots and animals, Science, 291, pp. 599-600, 2001.

[9] J. Zlatev, C. Balkenius, "Introduction: Why "epigenetic robotics'?", Proc. First International Workshop on Epigenetic Robotics, Lund University Cognitive Studies, Volume 85, pp. 1-4, 2001.

[10] S. Y. Yoon, R. C. Burke, B. Blumberg, G. E. Schneider, "Interactive training for synthetic characters, Proc. AAAI/IAAI, pp. 249-254, 2000

[11] F. Kaplan, P.-Y. Oudeyer, E Kubinyi, A. Miklosi, "Robotic clicker training", Robotics and Autonomous Systems, 38(3-4), pp. 197-206, 2002.

[12] K. Dautenhahn, A. Billard, "Studying Robot Social Cognition Within a Developmental Psychology Framework", Proc. Third European Workshop on Advanced Mobile Robots, pp. 187-194, 1999.

[13] P. Maes, "Agents that reduce work and information overload", In J. M. Bradshaw (Ed.), "Software Agents". AAAI Press/The MIT Press, pp. 145-164, 1997.

[14] B. Shneiderman, "Direct manipulation versus agents - Paths to predictable, controllable, and comprehensible interfaces", In J. M. Bradshaw (Ed.), "Software Agents". AAAI Press, pp. 97-106, 1997.

[15] K. Dautenhahn, C. L. Nehaniv (2000), "Living with Socially Intelligent Agents: A Cognitive Technology View". In K. Dautenhahn (Ed.), "Human Cognition and Social Agent Technology", John Benjamins Publishing Company, pp. 415-426, 2000.

[16] R. Coppinger, L. Coppinger, "Dogs - A Startling New Understanding of Canine Origin, Behavior \& Evolution", Scribner, 2001.

[17] R. Trappl, P. Petta (Eds.), "Creating Personalities for Synthetic Actors", Springer 1997.

[18] K. Dautenhahn, I. Werry, T. Salter, R. te Boekhorst, "Towards adaptive autonomous robots in autism therapy: Varieties of interactions", Proc. IEEE CIRA'03, pp. 577-582, 2003.

[19] T. Salter, R. te Boekhorst, K. Dautenhahn, "Detecting and analysing children's play styles with autonomous mobile robots: A case study comparing observational data with sensor readings" Proc. IAS-8 pp. 61-70, 2004.

[20] K. Dautenhahn, B. Ogden, T. Quick, "From embodied to socially embedded agents - Implications for interaction-aware robots". Cognitive Systems Research, 3(3), pp. 397-428, 2002. 